\title{
EDITORIAL
}

\section{A note of thanks}

\author{
Albert Hofman
}

Published online: 29 November 2013

(C) Springer Science+Business Media Dordrecht 2013

The editor-in-chief, editors, members of the editorial board and publisher of the European Journal of Epidemiology wish to express their sincere gratitude to the referees of the articles considered for the 2013 volume of the Journal.

Victor Abraira
Hisashi Adachi
Antonio Agudo
Olof Akre
Annick Alperovitch
André Amaral
Masoud Amiri
Philip Autier
Katarina Bälter
David Batty
Gundula Behrens
Gail Bell
Ingvar Bergdahl
Manuela Bergmann
Joline Beulens
Eric Boersma
Marike H. Boezen
Matteo Bonzini
Ralph Brinks
Annie Britton
Karin Broberg Palmgren
Linda Broer
Bert Brunekreef
Guy Brusselle
Gabrielle Buitendijk
Lex Burdorf
Stephen Burgess
Remi Charrel

A. Hofman $(\square)$

Rotterdam, The Netherlands

e-mail: a.hofman@erasmusmc.nl

\author{
Natasha Chen \\ Yen-Ching \\ Henrik Christesen \\ Shu-Chun Chuang \\ Jan Willem Coebergh \\ Anna Conen \\ Nancy Cook \\ Andrew Czeizel \\ Anna Dahl \\ Jean Dallongeville \\ Jean Francois Dartigues \\ Inez De Beaufort \\ Renée de Bruijn \\ Jessica Kiefte de Jong \\ Harry de Koning \\ Abbas Dehghan \\ Cyrille Delpierre \\ Hassan Dib \\ Eric Duell \\ Christina Ellervik \\ Jean Philippe Empana \\ Artur Fedorowski \\ Janine Felix \\ Pietro Ferrari \\ Jane Ferrie \\ Rahime Bedir Findik \\ Oscar Franco \\ Josep Garre-Olmo
}


Audrey Gaskins

Jean Gaudart

Mirjam Geerlings

Linda Geiss

Johanna Geleijnse

Akhgar Ghassabian

Reeta Gobin

Monica Guxens

Maria Hagströmer

Susan Heckbert

Jan Heeringa

Ningqi Hou

Frank Hu

Elina Hypponen

Mohammad Ikram

Hazel Inskip

Lianna Ishihara

Lisa Iversen

Rune Jacobsen

Pauline Jansen

Mazda Jenab

Magnus Kaijser

Maryam Kavousi

Helle Kieler

Leslie Klevay

Marija Knezevic-Pogancev

Roger Koenker

Michael Koller

Vladimir Kostic

Stephanie Kovalchik

Anton Kunst

Setor Kunutsor

Marina Kvaskoff

Andreas Kyrozis

Fiona L.R. Williams

Joep Lagro

Timothy Lash

Jari Laukkanen

Maarten Leening

Kuanrong Li

Bruno Lina

Greger Lindberg

Maja Lisa Løchen

Marie Lof

Jonas Ludvigsson

Annemarie Luik

Reedik Magi

Pedro Marques-Vidal

Reginald Marsh

Miguel-Angel Martinez-Gonzalez

Cecilia Mattisson

Claudia Meinke

Olli Miettinen
Saria Mirza

Sunni Mumford

Øyvind Næss

Ashley Naimi

Jennifer Hairong Nan

Katharina Nimptsch

Sam Norton

Ute Nöthlings

Wilma Nusselder

Ola Olén

Jorn Olsen

Trevor Orchard

Philip Orfanos

Jan Erik Otterstad

Simon Overland

Frederick Palm

Chenwei Pan

Sanne Peters

Christina Petersen

Eleni Petridou

Andreas Pettersson

Arne Pfeufer

Jerry Polesel

Charles Poole

Nancy Potischman

John Powles

Inga Prokopenko

Hein Raat

Inayat-Ur Rahman

Lorenzo Richiardi

Zoltán Rihmer

Sabine Rohrmann

Michael Rosenberg

Frits Rosendaal

Kenneth Rothman

Jean Bernard Ruidavets

Jonathan Samet

Amand Schmidt

Robert Scott

Diego Serraino

Alessandro Serretti

Susan Shortreed

Eric Sijbrands

Anders Skrondal

Harold Snieder

Meir J. Stampfer

Regine Steegers

Olof Stephansson

Ewout Steyerberg

Camilla Stoltenberg

Bruno Stricker

Reijo Sund

Dirk Taeger 
Naoko Tajima

Andrew Thorne-Lyman

Laura Thornton

Nic Timpson

Dimitrios Trichopoulos

Federica Turati

Linda Valeri

Aad van der Lugt

Carla H.van Gils

Hans C. van Houwelingen

Frank van Rooij

John C. van Swieten

Tyler VanderWeele

André L. M. Verbeek
Virginie Verhoeven

Meike Vernooij

Paolo Vineis

Jesus Vioque

Loes Visser

Nikolaus Weiskopf

Andreas Wienke

Klas Winell

Karin Wirdefeldt

Kristine Yaffe

Hanieh Yaghootkar

Wan-Shui Yang

Noah Zaitlen

Lisette Zuurbier 